\title{
Evidenziazione molecolare di resistenza di Mycobacterium tuberculosis complex a rifampicina e isoniazide
}

\section{Gian Lorenzo Molinari, Anna Camaggi, Stefano Andreoni, Vesselina Kroumova, Ilaria Crespi, Stefania Grasso, Michela Zignani, Giacomo Fortina}

Laboratorio di Microbiologia e Virologia

Az. Ospedaliera "Maggiore della Carità"- Novara

Molecular detection of resistance of M. tuberculosis complex to isoniazid and rifampin

Key words: Mycobacterium tuberculosis complex, resistance to isoniazid and rifampin

\section{SUMMARY}

From June 2004 to June 2005, a total of 58 mycobacterial isolates were recovered from inpatients admitted to the "Ospedale Maggiore" in Novara, Italy. Most of the above strains were from respiratory secretions. Among the 32 isolates identified as Mycobacterium tuberculosis complex (MTC), susceptibility testing to streptomycin, isoniazid, rifampin, ethambutol and pyrazinamide was subsequently performed by using the MGIT 960 system. Starting from positive cultures, the Genotype MTBDR “Arnika” test, based on target amplification and stripbased reverse hybridization was also carried out.

In addition to the MTC identification, the test can investigate the resistance to both rifampin (by molecular analysis of the rpoB region) and isoniazid (by detecting mutations at the resistance domain of katG gene).

The results were compared with those obtained by conventional liquid medium susceptibility testing. Full agreement with both drugs was obtained on all the tested isolates.

In conclusion, the GenoType MTBDR assay was found to enable a very rapid resistance detection of the most important anti-tubercular drugs. These data are important to set up an effective therapy and consequent disease control.

\section{INTRODUZIONE}

Il ritorno della tubercolosi ha fatto sì che venissero affinati i test tradizionali e venissero sviluppati nuovi test in biologia molecolare che, in caso di sospetto clinico, potessero offrire una risposta sempre più rapida e affidabile (6).

Il dato più preoccupante di questi ultimi anni deriva dalla comparsa, anche in Italia, di ceppi di $M$. tuberculosis complex resistenti a uno o più farmaci antimicobatterici (4) compromettendo con ciò la buona riuscita di un trattamento antitubercolare.

Lo sviluppo di farmacoresistenze in M. tuberculosis complex è il risultato di mutazioni genetiche casuali localizzate in particolari geni (14).

Le mutazioni che predominano nei ceppi di $M$. tuberculosis complex rifampicina-resistenti sono localizzate per il $95 \%$ in una regione denominata "regione core" del gene rpoB $(1,12)$.

$\mathrm{Al}$ contrario, le mutazioni che conferiscono resistenza all'isoniazide sono localizzate in diversi geni e regioni: approssimativamente il 50-95\% dei ceppi isoniazide-resistenti presenta mutazioni nel codone 315 del gene katG, (11) il 20-35\% pos- siede mutazioni nella regione regolatoria ihnA (5) mentre il $10-15 \%$ presenta mutazioni nella regione intergenica ahpC-oxyR spesso associate alle mutazioni in katG fuori dal codone (9).

\section{MATERIALI E METODI}

Nel periodo giugno 2004-giugno 2005 sono stati isolati presso il Laboratorio di Microbiologia dell'Ospedale Maggiore di Novara n. 58 ceppi di micobatteri provenienti in particolare da materiale respiratorio. Le colture sono state sempre effettuate utilizzando un terreno liquido fluorimetrico (Bactec MGIT 960) associato ad un terreno solido tradizionale (Lowenstein-Jensen).

Dei 58 ceppi, 32 sono stati identificati come Mycobacterium tuberculosis complex mediante l'utilizzo di sonde genetiche GeneProbes-San Diego California (bioMérieux) (10).

Nei confronti dei 32 ceppi di $M$. tuberculosis complex è stato poi effettuato l'antibiogramma utilizzando Bactec MGIT 960 (Becton Dickinson) che consente in un periodo medio di 5 giorni dalla crescita primaria di determinare la sensibilità 0 resistenza del micobatterio nei confronti di strep- 
tomicina, isoniazide, rifampicina, etambutolo in normali provette MGIT antibiotate, nonché pirazinamide con l'utilizzazione di particolari provette MGIT acidificate $(7,13)$.

Gli stessi ceppi sono stati saggiati anche con GenoType MTBDR della Ditta "Arnika", un test che evidenzia in poche ore l'eventuale resistenza a rifampicina e isoniazide.

La procedura è suddivisa in tre fasi successive:

1) estrazione del DNA mediante termoblocco a $95^{\circ} \mathrm{C}$ per 20 minuti e bagno ultrasonico per 15 minuti, partendo direttamente dal flacone MGIT positivo o dalle colonie sviluppatesi su terreno solido

2) amplificazione multiplex utilizzando una miscela di primers biotilinati specifici, nucleotidi, dimetil-sulfossido, cloruro di magnesio e Taq polimerasi

3) ibridazione inversa durante la quale $20 \mu \mathrm{ldel}$ campione, amplificato e denaturato a temperatura ambiente, vengono messi a contatto nella apposita vaschetta con una strip coattata con sonde specifiche.

Questo test, della durata complessiva di circa cinque ore, oltre alla conferma di identificazione del ceppo, permette contemporaneamente di determinare la resistenza alla rifampicina tramite l'analisi molecolare mediante sonde, dell'area di resistenza del gene rpoB (che codifica per la subunità $\beta$ dell'RNA polimerasi), e all'isoniazide andando a testare la regione di resistenza del gene katG (che codifica per l'enzima catalasi perossidasi) (2, $3,8)$.

Sono stati poi saggiati, sempre con l'utilizzazione del GenoType MTBDR "Arnika", due ceppi controllo di M. tuberculosis complex, ceppi inviatici dal "Centro di Riferimento per le Micobatteriosi" Villa Marelli di Milano, che avevano la caratteristica di essere l'uno resistente a isoniazide e l'altro a isoniazide + rifampicina.

\section{Interpretazione delle strip}

Nella figura I è rappresentata una striscia di interpretazione nella quale sono evidenziate le disposizioni delle varie sonde coattate.

La sonda rpoB Uni analizza una regione specifica del gene rpoB e deve sempre sviluppare una colorazione. Le cinque sonde rpoB wild type (rpoB WT 1-5) comprendono le principali zone di resistenza alla rifampicina. Le 4 sonde di mutazione rpoB permettono di determinare le mutazioni D516V, H526Y, H526D e S531L all' origine della resistenza alla rifampicina stessa (figura II). Lo sviluppo di colorazione nelle zone di reazione permette di documentare la presenza del gene rpoB wild type o mutato. Lo sviluppo di tutte le zone rpoB WT 1-5 indica l'assenza di mutazioni degli acidi nucleici nelle regioni esaminate. In caso di mutazione di un acido nucleico, il rispettivo amplicone non si può legare alla corrispondente sonda wild type. L'assenza di segnale in una zona di reazione wild type indica resistenza verso la rifampicina. Se la resistenza trova la sua origine in corrispondenza di una delle 4 principali mutazioni del gene rpoB, si svilupperà un segnale in corrispondenza della rispettiva sonda di mutazione e la sonda wild type corrispondente non svilupperà colorazione.

La sonda katG Uni analizza una regione specifica del gene katG e deve sempre sviluppare una colorazione. La sonda katG wild type analizza la regione di resistenza all'isoniazide in posizione 315 .

Lo sviluppo di bande colorate nelle zone di reazione permette di evidenziare la presenza del gene katG wild type o mutato. Se la sonda wild type (katG WT) sviluppa un segnale, in assenza del segnale in corrispondenza delle sonde di mutazione katG (katG MUT 1/2), il ceppo testato è sensibile all'isoniazide. Se è presente una mutazione in posizione 315 , l'amplicone prodotto non ibrida con la sonda wild type. L'assenza di segnale indica dunque che il ceppo testato è resistente all'isoniazide.

\section{RISULTATI}

Il confronto tra i dati ottenuti con il kit GT MTBDR e quelli successivamente forniti dall'antibiogramma effettuato con il tradizionale BACTEC MGIT, per quanto riguarda isoniazide e rifampicina, ha evidenziato una totale sovrapponibilità di risultati.

Dei 32 casi di $M$. tuberculosis complex presi in considerazione, 29 sono risultati sensibili a isoniazide e rifampicina utilizzando entrambi i metodi, così come le resistenze riferite agli altri 3 ceppi, immediatamente segnalate dal sistema GT MTBDR sono successivamente state confermate dall'antibiogramma tradizionale.

Anche i 2 ceppi di controllo resistenti l'uno a rifampicina e l'altro a isoniazide + rifampicina, hanno trovato immediata conferma dal test GT MTBDR.

Nella figura III sono rappresentate le strisce riferite ai 3 ceppi di $M$. tuberculosis complex resistenti a isoniazide + rifampicina (figura III, striscia 1, 2, 3), la striscia riferita ai 29 casi sensibili a isoniazide e rifampicina (viene rappresentata solo una delle 29 strisce tutte uguali fra di loro) (figura III, striscia 4), nonché le strisce riferite ai due controlli di qualità, uno resistente a isoniazide + rifampicina (figura III, striscia 5) e l'altro resistente alla sola isoniazide (figura III, striscia 6). 


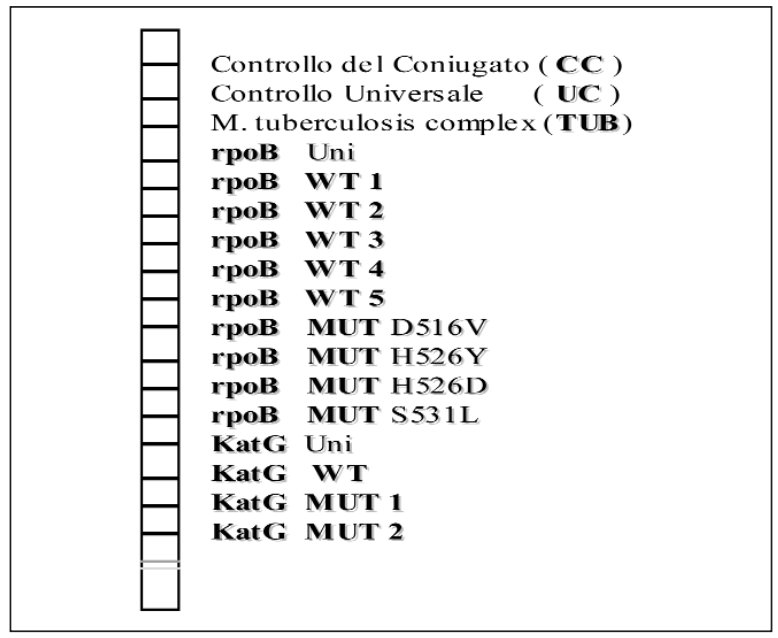

Figura I. Striscia di interpretazione.

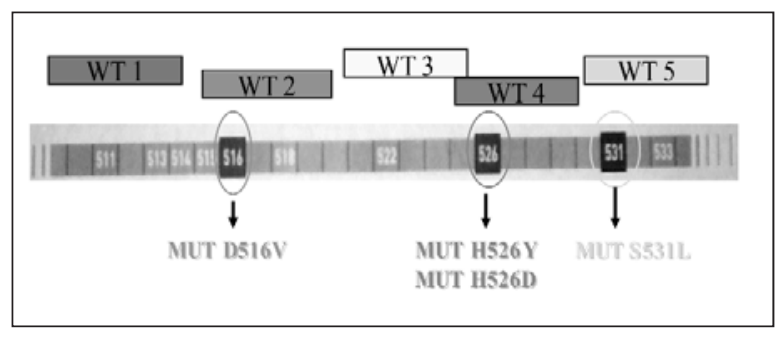

Figura II. Regione rifampicina resistenza del gene rpoB. I numeri precisano la posizione degli aminoacidi (codoni) per le mutazioni rilevabili attraverso la strip.

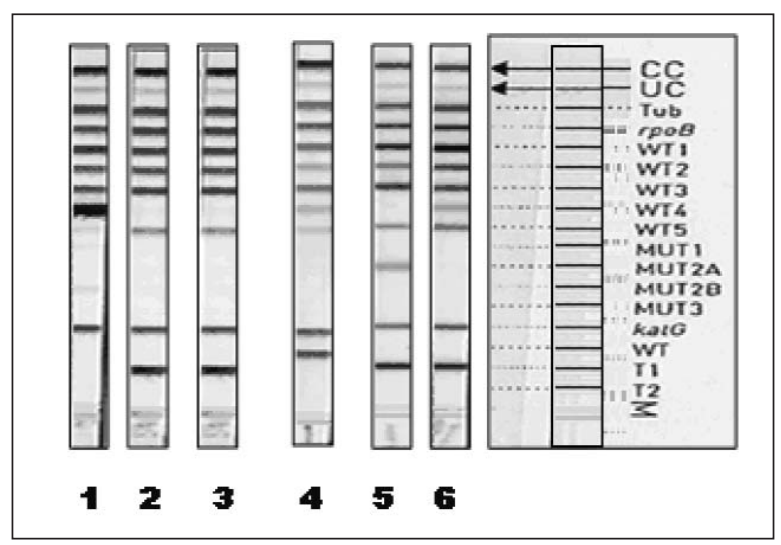

Figura III.

\section{DISCUSSIONE}

Pur considerando questi dati preliminari e meritevoli di ulteriori approfondimenti (in particolare sarebbe interessante un'analisi di sequenziamento per confermare le mutazioni che hanno causato la resistenza o evidenziare eventuali altre mutazioni che potrebbero portare allo stesso risultato) possiamo affermare che queste nuove metodiche permettono una significativa riduzione dei tempi di risposta consentendo di attuare una terapia sempre più efficace e tempestiva.

Non bisogna però dimenticare che il GenoType
MTBDR, analizza la sequenza di acidi nucleici e non la sequenza di aminoacidi: in particolare il test preso in considerazione evidenzia solamente le resistenze di $M$. tuberculosis complex che trovano la loro origine nelle regioni $\mathrm{rpoB}$ e $\mathrm{katG}$, anche se tali resistenze sono la stragrande maggioranza; pertanto le resistenze che hanno per origine mutazioni situate in altri geni, o le resistenze nei confronti di streptomicina, etambutolo e pirazinamide non sono per questo motivo evidenziate dal test.

I dati in nostro possesso, pur essendo ancora numericamente poco rilevanti, ci consentono però di poter affermare che le resistenze segnalate da Geno Type MTBDR vengono poi puntualmente confermate dall'antibiogramma tradizionale, per cui il fatto di poter immediatamente segnalare al medico curante la resistenza nei confronti di isoniazide e rifampicina viene comunque ritenuto un fatto assolutamente importante al fine di impostare tempestivamente una corretta terapia antitubercolare.

Infine è giusto sottolineare che il costo elevato per singola determinazione non rende evidentemente semplice e scontata una sua applicazione in ogni situazione anche se, quando si deve valutare il rapporto costo-beneficio di un nuovo test, è necessario considerare anche altri parametri oltre al semplice costo del reagente. In base a questo, il test in esame sembra essere conveniente, anche economicamente, quando lo si utilizzi là dove è alto il rischio di insorgenza di malattia o quando si ritiene possibile l'instaurarsi di resistenze che potrebbero rendere inefficace la prima scelta terapeutica.

\section{BIBLIOGRAFIA}

1. Bartfai Z, Somoskovi A, Kodmon C, et al. Molecular characterization of rifampin-resistant isolates of Mycobacterium tuberculosis from Hungary by DNA sequencing and the line probe assay. J Clin Microbiol 2001; 39: 3736-9.

2. Cavallerio P, Piana P, Matinato C, et al. Molecular detection of rifampicin and isoniazid mutation in multiresistant $M$. tuberculosis strains isolated in Italy. 2004; 26 th Annual Congress of the Society of Mycobacteriology.

3. Hillemann D, Weizenegger M, Kubica T, Richter E, Niemann S. Use of the Genotype MTBDR Assay for Rapid Detection of Rifampin and Isoniazid Resistance in Mycobacterium tuberculosis complex isolates. J Clin Microbiol 2005; 43: 3699-703.

4. Migliori GB, Centis R, Fattorini L, et al. Mycobacterium tuberculosis complex drug resistance in Italy. Emerg Infect Dis 2004 Apr; 10(4): 752-3.

5. Piatek AS, Telenti A, Murray R, et al. Genotypic analysis of Mycobacterium tuberculosis in two distinct populations using molecular beacons: implications for rapid susceptibility. Antimicrob Agents Chemother 2000; 44(1): 103-10. 
6. Piersimoni C, Nista D, Galassi M, Cimarelli ME, Tubali A. Clinical suspicion as a primary guidance to use commercial amplification tests for rapid diagnosis of pulmonary tuberculosis. Diagn Microbiol Infect Dis 2005 Nov; 53(3): 195-200.

7. Scarparo C, Ricordi P, Ruggiero G, Piccoli P. Evaluation of the fully automated BACTEC MGIT 960 System for testing susceptibility of Mycobacterium tuberculosis to pyrazinamide, streptomycin, isoniazid, rifampin and ethanbutol and comparison with the radiometric BACTEC 460 TB method. J Clin Microbiol 2004; 42: 1109-14.

8. Sojni H, Maktnen J, Marttila H, Marjamakt M. Evaluation of a novel strip test genotype MTBDR for the detection of multi-drug resistant Mycobacterium tuberculosis. 2004; 26 th Annual Congress of the Society of Mycobacteriology.

9. Srrevatsan S, Pan X, Zhang Y, Deretic V, Musser JM. Analysis of the oxyR-ahpC region in isoniazid-resistant and -susceptible Mycobacterium tuberculosis complex organisms recovered from discased humans and animals in diverse localities. Antimicrob Agents Chemother 1997; 41: 600-6.

10. Stockman L. DNA probes for identification of mycobacteria in Isemberg HD. Clinical microbiology procedures handbook 3.15.1-3.15.4 1992 American society for microbiology, Washington.

11. Telenti A, Honorè N, Bernasconi C, et al. Genotyping assessment of isoniazid and rifampin resistance in Mycobacterium tuberculosis: a blind study at reference laboratory level. J Clin Microbiol 1997; 35: 71923.

12. Telenti A, Imboden P, Marchesi F, et al. Detection of rifampicin-resistance mutations in Mycobacterium tuberculosis. Lancet 1997; 341: 647-50.

13. Tortoli E, Benedetti M, Fontanelli A, Simonetti MT. Evaluation of automathed BACTEC MGIT 960 System for testing susceptibility of Mycobacterium tuberculosis to four major antituberculous drugs: comparison with the radiometric BACTEC 460 TB method and the agar plate method of proportion. $\mathrm{J}$ Clin Microbiol 2002; 40: 607-10.

14. Zhang Y, Telenti A. Genetics of drug resistance in Mycobacterium tuberculosis. In Hatfull GF, Jacobs WR-Jr Molecular genetics of mycobacteria. Washington (DC): ASM Press; 2000 p 235-54.

Gian Lorenzo MOLINARI

Laboratorio di Microbiologia e Virologia Ospedale Maggiore della Carità Corso Mazzini 18 - 28100 - Novara Tel. 0321 3733595; Fax 03213733588 E-mail: gianlorenzo.molinari@maggioreosp.novara.it 